\title{
An Enhanced Gabor Filter-Based Segmentation Algorithm for Fingerprint Recognition Systems
}

\author{
Fernando Alonso-Fernandez, Julian Fierrez-Aguilar, Javier Ortega-Garcia \\ Biometrics Research Lab.- ATVS, Escuela Politecnica Superior \\ Universidad Autonoma de Madrid, Spain \\ \{fernando.alonso, julian.fierrez, javier.ortega $\} @$ uam.es
}

\begin{abstract}
An important step in fingerprint recognition is the segmentation of the region of interest. In this paper, we present an enhanced approach for fingerprint segmentation based on the response of eight oriented Gabor filters. The performance of the algorithm has been evaluated in terms of decision error trade-off curves of an overall verification system. Experimental results demonstrate the robustness of the proposed method.
\end{abstract}

\section{Introduction}

Due to its permanence and uniqueness, fingerprints are widely used in many personal identification systems. Fingerprints are being increasingly used not only in forensic environments, but also in a large number of civilian applications such as access control or on-line identification [1].

Fingerprint segmentation consists in the separation of the fingerprint area (foreground) from the background [2]. This is useful to avoid subsequent extraction of fingerprint features in the background, which is a noisy area. Using a global or local thresholding method for segmentation is not very effective and more robust segmentation techniques are commonly used. These techniques exploit the existence of an oriented periodical pattern in the foreground, and a nonoriented isotropic pattern in the background:

- The method described in [3] is based on the local certainty level of the orientation field, which is computed using the intensity gradient of the image. Those $16 \times 16$ pixel blocks in which the certainty level is higher than a given threshold are considered as foreground blocks.

- In [4] the average gradient on each block is computed, which is expected to be high in the foreground (ridgevalley variations) and low in the background.

- In [5], other parameters (gradient coherence, gray intensity mean and variance) are also used in the segmentation decision. A morphological postprocessing is also performed in order to fill the remaining holes in the foreground and/or in the background. This method is very accurate but involves high computational burden.

- The technique presented in [6] relies on the gradient and results in lower computational burden. It computes the gray level variance across the normal direction of the orientation field, which is expected to be high in presence of ridge-valley variation and low in the presence of noise. This method is implemented in other fingerprint verification systems as well [7].

- The segmentation technique presented in [8] is based on Gabor filters. It computes the response of eight oriented Gabor filters to determine whether a block belongs to the foreground or to the background.

In [8], it is shown that when good quality images are considered, both gradient- and Gabor-based methods produce similar results, but Gabor filter-based methods are faster than gradient-based approaches. In the present work, an enhanced Gabor filter-based approach is presented. Our method obtains higher foreground size and considerably lower size of the background region within the foreground, thus recovering blocks with minutiae and valid not well defined zones. The proposed method is evaluated in terms of DET curves [9] using a fingerprint database of mediumhigh image quality [10].

The standard Gabor filter-based segmentation strategy from which we have built our enhanced method is summarized in Sect. 2. The proposed method is described in Sect. 3. The fingerprint recognition system used to evaluate this method is described in Sect. 4. Experiments and results are given in Sect. 5. Conclusions are finally drawn in Sect. 6.

\section{Gabor Filter-Based Segmentation}

An even symmetric Gabor filter has the following general form in the spatial domain [11]:

$$
h(x, y, \theta, f)=\exp \left\{-\frac{1}{2}\left[\frac{x_{\theta}^{2}}{\sigma_{x}^{2}}+\frac{y_{\theta}^{2}}{\sigma_{y}^{2}}\right]\right\} \cos \left(2 \pi f x_{\theta}\right)
$$




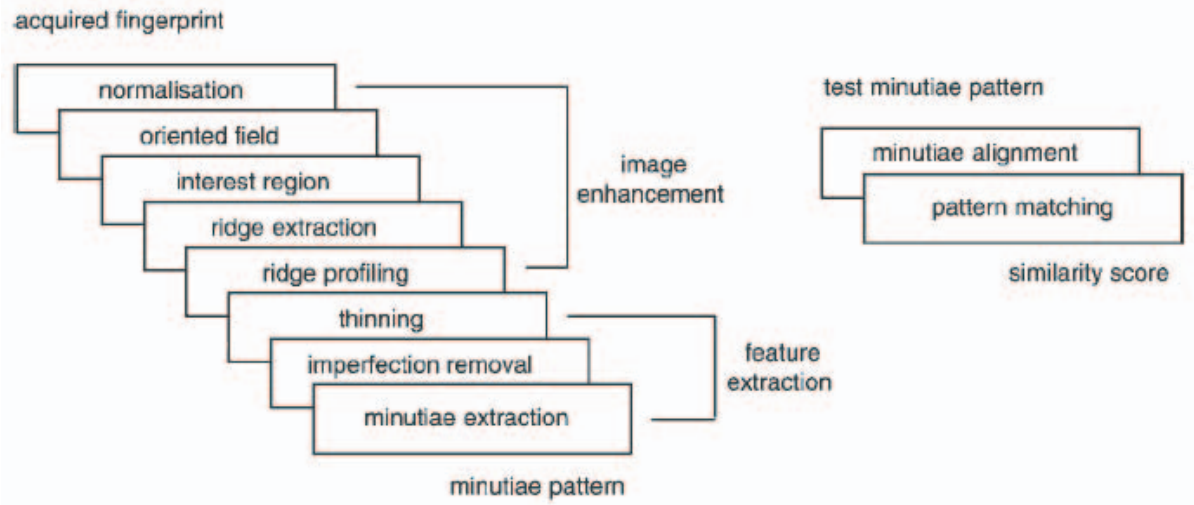

Figure 1. Block diagram architecture of the automatic fingerprint verification system [7].

where $x_{\theta}=x \cos \theta+y \sin \theta$, and $y_{\theta}=-x \sin \theta+y \cos \theta$.

This filter consist of a Gaussian envelope (of parameters $\sigma_{x}$ and $\sigma_{y}$ ) modulated by a sinusoid of frequency $f$ along the direction of the $x_{\theta}$ axis. The angle $\theta$ allows to rotate the direction of the response. Since local ridge structures of fingerprints can be modelled as oriented sinusoids along a direction normal to the local ridge orientation [12], this filter performs very well in segmenting oriented ridge zones and noisy non-oriented background zones. The frequency $f$ can be set as the inverse of the average inter-ridge distance. The value of $\theta$ is given by $\theta_{k}=\pi(k-1) / m, k=1, \ldots, m$, where $m$ denotes the number of orientations ( $m=8$ in this work).

For each image block of size $W \times W$ centered at $(X, Y)$, with $W$ even, we extract the magnitude Gabor feature [8] as follows for $k=1, \ldots, m$ :

$$
\begin{gathered}
g\left(X, Y, \theta_{k}, f, \sigma_{x}, \sigma_{y}\right)= \\
\left|\sum_{x_{0}=-W / 2}^{(W / 2)-1} \sum_{y_{0}=-W / 2}^{(W / 2)-1} I\left(X+x_{0}, Y+y_{0}\right) h\left(x_{0}, y_{0}, \theta_{k}, f, \sigma_{x}, \sigma_{y}\right)\right|
\end{gathered}
$$

where $I(x, y)$ denotes the gray level of the pixel $(x, y)$.

As a result, we obtain $m$ Gabor features for each $W \times W$ block of the image. In blocks with ridge pattern, the values of one or several Gabor features will be higher than the others (those values whose filter angle is similar to the ridge angle of the block). Alternatively, for noisy non-oriented background blocks, the $m$ values of the Gabor features will be similar. Therefore, the standard deviation $G$ of the $m$ Gabor features allows to segment foreground and background. If $G$ is less than a given threshold, the block is labelled as background block, otherwise the block is labelled as foreground block.

This technique has the following drawbacks: $(i)$ loss of precision occurs in the borders of the region of interest or in low contrast regions; and (ii) some valid regions which may contain important minutiae information are lost within the foreground.
The first problem can be solved as follows. A tolerance box is located around borders of the region of interest to discard minutiae found in this area, as they are not stable for recognition. The second problem is important since most fingerprint systems use these minutiae for recognition, so missing any of them has to be completely avoided.

\section{The Proposed Segmentation Method}

As mentioned above, the standard Gabor filter-based segmentation approach presents some problems. With the aim of coping with these problems, three modifications to the standard Gabor filter-based approach are proposed, namely: $(i)$ overlapping blocks, $(i i)$ ridge frequency computation, and (iii) heuristic constraints.

\subsection{Overlapping Blocks}

In our approach, we use blocks of size $W \times W$ with an overlapping of $W / 2$ pixels. As a result, we obtain 4 different Gabor features for each $W / 2 \times W / 2$ block of the image, which are then averaged (mean rule).

\subsection{Ridge Frequency Computation}

In the present work, we propose to estimate the local frequency field of the fingerprint and to use these estimated values as Gabor filter parameters, instead of considering the frequency as a constant value. The algorithm used to estimate the ridge frequency is described in [12].

\subsection{Heuristic Constraints}

In our approach, some additional heuristic constraints have been imposed in order to discard those blocks not suitable for the frequency estimation algorithm (i.e. whose $x$ signature [12] does not form a sinusoidal-shaped wave, such as background blocks or blocks where minutiae or singular points appear): ( $i$ ) blocks with luminosity lower than a given threshold in most of its $x$-signature points are discarded; (ii) the total number of peaks (maxima) plus valleys 


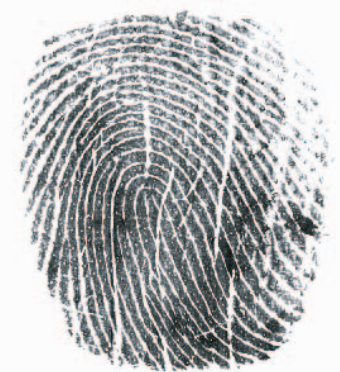

(a)

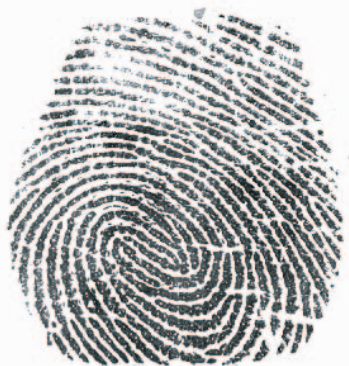

(b)

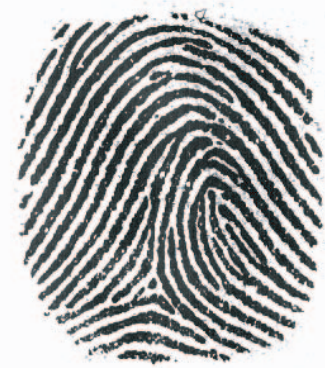

(c)

Figure 2. Sample images of the ATVS database

(minima) in the sinusoid represented by the $x$-signature has to be 3 or more; and (iii) the luminosity value of all peaks (maxima) of the $x$-signature has to be higher than a given threshold.

\section{Fingerprint Recognition System}

The architecture of the automatic fingerprint recognition system used in the experiments is shown in Fig. 1. It can be divided in four phases [7]: $(i)$ fingerprint image acquisition; (ii) image enhancement process; (iii) feature extraction from the enhanced image; and (iv) pattern matching process.

\subsection{Image enhancement}

The aim of this stage is to provide a high-quality image. Image imperfections may degrade the recognition system performance, making this image enhancement procedure necessary.

Regarding our system, the complete sequence of stages for image enhancement is: $(i)$ normalization; (ii) calculation of the orientation field; (iii) region of interest extraction; $(i v)$ ridge extraction; and $(v)$ ridge profiling. Details of each stage are explained in [7]. In order to compute the region of interest, we use the algorithms proposed in this paper.

\subsection{Feature extraction}

The sequence of processes to generate a reliable biometric pattern is [7, 13]: $(i)$ thinning of the reconstructed binary ridge structure achieved after image enhancement; $(i i)$ removal of all structure imperfections from the thinned image, and (iii) minutiae extraction.

\subsection{Pattern recognition}

Given two biometric patterns, namely query and enrolled patterns, the verification process is aimed to determining whether these fingerprint patterns have been produced by the same finger or not. The two patterns are aligned before fingerprint matching $[7,3]$. Then, a score is defined to measure the similarity (edit distance) between the two patterns. The elastic technique used permits certain adaptive spatial tolerance margin to compensate for the nonlinear elastic deformations $[7,3,13]$.

\section{Experiments}

\subsection{ATVS Database}

The 100SC Precise Biometrics scanner has been used to acquire a fingerprint database (ATVS database) [10]. It consists of 50 users, with 8 samples of the same finger per user, producing 500 dpi images of $300 \times 300$ pixels. As a result, $50 \times 8=400$ different fingerprint images are available. Acquisition process was manually supervised to ensure that all images have a minimum quality. In Figure 2 we can see some images of this database.

\subsection{Alternatives Tested}

In order to evaluate the contributions made in this paper to Gabor filter-based segmentation (i.e., overlapping blocks and ridge frequency estimation with heuristic constraints), the next alternatives have been tested: (1) using the standard segmentation algorithm described in Sect. 2; and (2) using the enhanced segmentation algorithm proposed in Sect. 3.

\subsection{Experimental Results}

Segmentation is based on thresholding the standard deviation $G$ of the $m$ Gabor features (Sect. 2). In this section, we present some experiments varying this threshold.

Verification tests have been performed as follows: $(i)$ the first sample of each finger is matched with the remaining 7 samples, resulting in $7 \times 50=350$ genuine accesses; and (ii) the first sample of each finger is matched with 7 randomly chosen samples of the remaining fingers, resulting in $7 \times 50=350$ impostor accesses.

The parameters used in the experiments are: $1 / f=7$ (average inter-ridge distance in 500 dpi fingerprint images), $\sigma_{x}=\sigma_{y}=4, m=8$ filters, and $W=16$ pixels. 


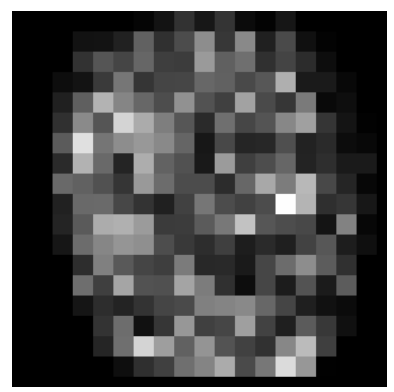

(a) Standard deviation $G$ (black color means zero value and white color means the maximum value)

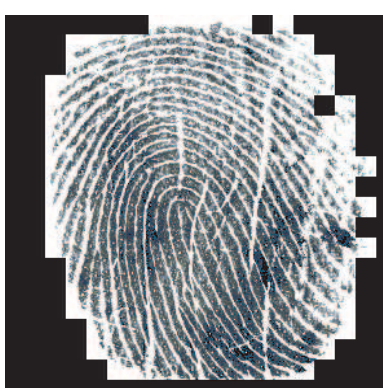

(b) $T=100$

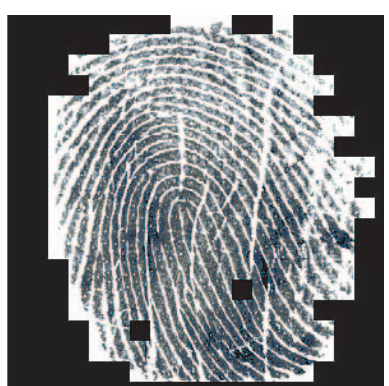

(c) $T=200$

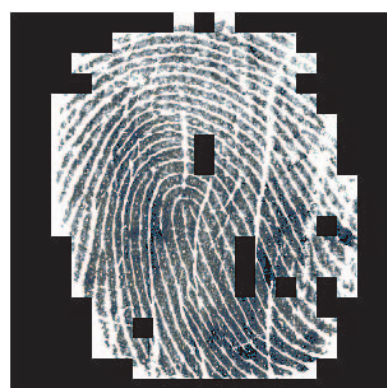

(d) $T=300$

Figure 3. Standard deviation $G$ of the $m$ Gabor features and resulting segmented area of the image shown in Fig. 2(a) using the standard segmentation algorithm for each of the segmentation thresholds.

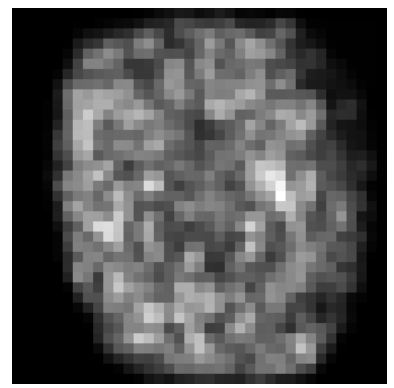

(a) Standard deviation $G$ (black color means zero value and white color means the maximum value)

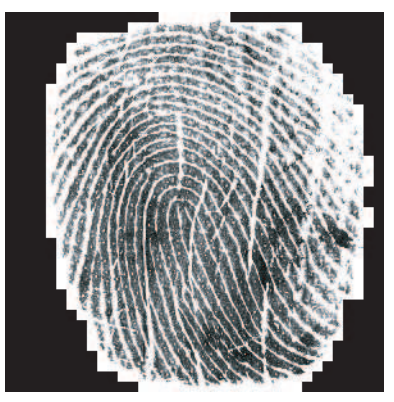

(b) $T=100$

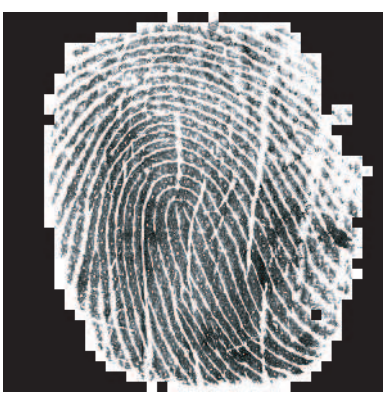

(c) $T=200$

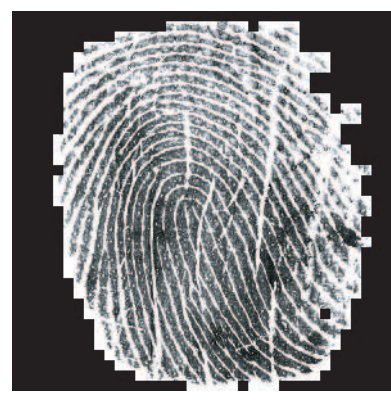

(d) $T=300$

Figure 4. Standard deviation $G$ of the $m$ Gabor features and resulting segmented area of the image shown in Fig. 2(a) using the enhanced segmentation algorithm proposed for each of the segmentation thresholds. 


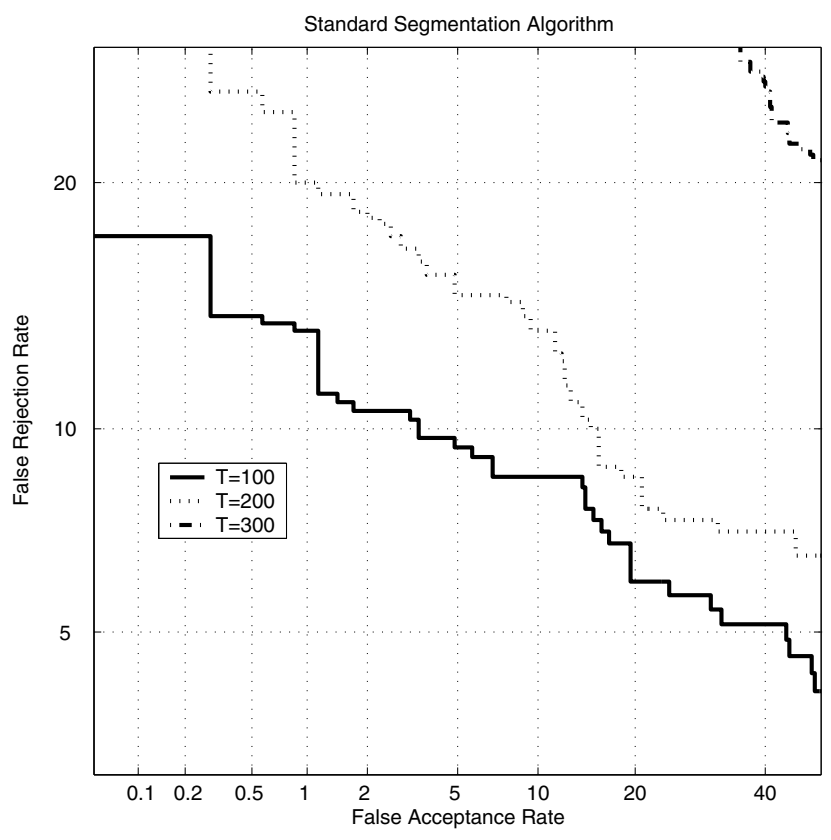

(a)

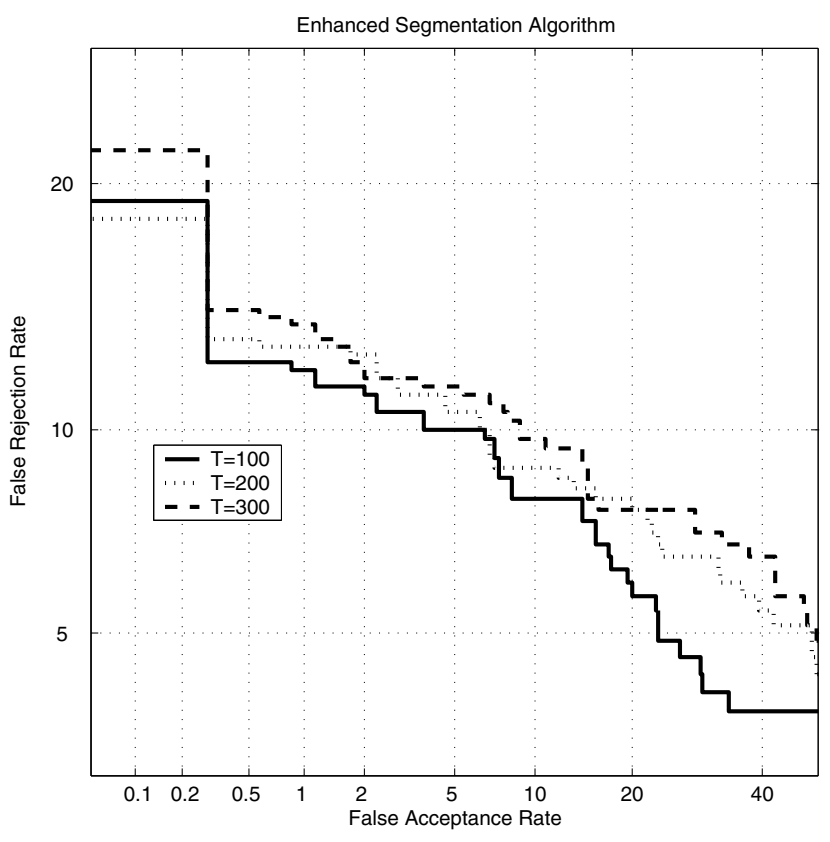

(b)

Figure 5. Verification performance of our minutiae-based fingerprint system on ATVS database using the standard (a) and proposed (b) segmentation algorithms for increasing segmentation threshold $T$

In Fig. 5, DET curves for each alternative indicated in Sect. 5.2 are shown varying the segmentation threshold $T$.

In Figs. 3-4 we can see the resulting segmented area of the image shown in Fig. 2(a) for each of the alternatives and the thresholds shown in Fig. 5. Standard deviation $G$ is also depicted.

Using the standard segmentation algorithm, valid regions are lost within the foreground (Fig. 3(b),3(c),3(d)). Using the proposed method, the number of lost regions are considerably lower (Fig. 4(b),4(c),4(d)). Moreover, when considering high quality images, this number tends to zero.

From Fig. 5, we can conclude that the proposed algorithm increases the robustness of our system since there is a wider range of segmentation thresholds where error rates remain almost constant. Using the standard segmentation algorithm dramatically increases FAR and FRR as we increase the segmentation threshold (Fig. 5(a)). Higher robustness is achieved with the enhanced segmentation algorithm, as DET curves in Fig. 5(b) are more concentrated. In addition, our enhanced algorithm produces lower error at low FRR.

\section{Conclusions and Future Work}

We have presented an enhanced Gabor filter-based fingerprint segmentation method which includes block overlapping and ridge frequency computation. This new approach overcome the problem of loss of valid regions within the foreground. The proposed overlap-based approach takes into account the presence of ridge pattern in the neighbor- hood of the block under consideration, resulting in a correct segmentation decision for those valid blocks with minutiae.

The performance of the proposed and existing algorithms have been evaluated in terms of DET curves using a database with medium-high quality fingerprint images. Experimental results show that the proposed enhanced algorithm provides higher robustness to the overall verification system.

Our future work is oriented to evaluate the performance of our system with fingerprint image sets of different quality.

\section{Acknowledgements}

This work has been supported by the TIC2003-08382C05-01 project of the Spanish Ministry of Science and Technology. F. A.-F. and J. F.-A. thank Consejeria de Educacion de la Comunidad de Madrid and Fondo Social Europeo for supporting their $\mathrm{PhD}$ studies.

\section{References}

[1] A. K. Jain, A. Ross, and S. Prabhakar, "An introduction to biometric recognition," IEEE Transactions on Circuits and Systems for Video Technology, vol. 14, pp. 4-20, Jan. 2004.

[2] D.Maltoni, D.Maio, A.Jain, and S.Prabhakar, Handbook of Fingerprint Recognition. Springer, New York, 2003. 
[3] A. K. Jain, L. Hong, S. Pankanti, and R. Bolle, "An identity authentication system using fingerprints," Proc. IEEE, vol. 85, pp. 1365-1388, Sept. 1997.

[4] Dario Maio and Davide Maltoni, "Direct gray-scale minutiae detection in fingerprints," IEEE Trans. on Pattern Analysis and Machine Inteligence, vol. 19, pp. 27-40, Jan. 1997.

[5] A. Bazen and S. Gerez, "Segmentation of fingerprint images," in Proc. Workshop on Circuits Systems and Signal Processing, ProRISC 2001, pp. 276-280.

[6] B. Mehtre, "Fingerprint image analysis for automatic identification," Machine Vision and Applications, vol. 6, pp. 124-139, 1993.

[7] D. Simon-Zorita, J. Ortega-Garcia, J. Fierrez-Aguilar, and J. Gonzalez-Rodriguez, "Image quality and position variability assessment in minutiae-based fingerprint verification," IEE Proceedings - Vis. Image Signal Process., vol. 150, pp. 402-408, Dec. 2003.

[8] LinLin Shen, Alex Kot, and WaiMun Koo, "Quality measures of fingerprint images," in Proc. 3rd Audio and Video-Based Person Authentication, AVBPA 2001, pp. 266-271.

[9] A. Martin, G. Doddington, T. Kamm, M. Ordowski and M. Przybocki, "The DET curve in assessment of decision task performance," in Proc. of ESCA Eur. Conf. on Speech Comm. and Tech., EuroSpeech 1997, pp. 1895-1898.

[10] S. Cruz-Llanas, J. Gonzalez-Rodriguez, D. SimonZorita, and J. Ortega-Garcia, "Minutiae extraction scheme for fingerprint recognition systems," in Proc. Int. Conf. on Image Processing, ICIP 2001, pp. 254257.

[11] A. K. Jain, S. Prabhakar, L. Hong, and S.Pankanti, "Filterbank-based fingerprint matching," IEEE Transactions on Image Processing, vol. 9, pp. 846-859, May 2000.

[12] Lin Hong, Yifei Wan, and Anil Jain, "Fingerprint imagen enhancement: Algorithm and performance evaluation," IEEE Trans. on PAMI, vol. 20, pp. 777-789, Aug. 1998.

[13] A. Jain, L. Hong, and R. Bolle, "On-line fingerprint verification," IEEE Trans on PAMI, vol. 19, pp. 302314, April 1997. 